Wijnhoven, T.; Tant, J.; Deconinck, G., "Inverter modelling techniques for protection studies," in 3rd IEEE International Symposium on Power Electronics for Distributed Generation Systems (PEDG), Aalborg, Denmark, 25-28 June 2012, pp.187-194.

doi: 10.1109/PEDG.2012.6253999

URL: http://ieeexplore.ieee.org/stamp/stamp.jsp?tp=\&arnumber=6253999\&isnu mber $=6253967$

(c) 2012 IEEE. Personal use of this material is permitted. Permission from IEEE must be obtained for all other users, including reprinting/ republishing this material for advertising or promotional purposes, creating new collective works for resale or redistribution to servers or lists, or reuse of any copyrighted components of this work in other works.

(below follows the accepted version) 


\title{
Inverter Modelling Techniques for Protection Studies
}

\author{
Thomas Wijnhoven, Jeroen Tant and Geert Deconinck \\ ELECTA, Department of Electrical Engineering, KU Leuven, Belgium
}

\begin{abstract}
Traditional fault calculation techniques are not capable of accurately modelling the short-circuit behaviour of inverter interfaced distributed generation (IIDG). As the share of IIDG continues to rise, more accurate fault simulations are required. This paper gives an overview of fault calculation techniques described in literature. All techniques are evaluated from the viewpoint of protection studies in medium and low voltage grids with a high share of IIDG. The averaged value modelling technique is selected. The merits of this technique are illustrated in a Simulink/PLECS simulation environment, where averaged value models are compared to full time-domain switched models in terms of simulation time and accuracy.
\end{abstract}

Index Terms-power system protection, distributed power generation, inverter, simulation, dynamic-average value modelling

\section{INTRODUCTION}

In order to achieve the full potential of renewable energy, local opportunities to generate electric power should be optimised. However, to guarantee cost effective solutions, distributed energy sources (DER) should be connected to the local distribution grid wherever possible, as grid expansions are often very costly. Given the ambitious European goals for renewable energy, large scale integration of DER in the distribution grids is expected. In areas with a high share of DER, utilities are already experiencing protection challenges. Studying the impact of this large scale integration on the distribution grid, and developing mitigating measures, requires reliable simulation models for all types of DER. These models are readily available for induction and synchronous generators, but not for inverter interfaced distributed generation (IIDG).

In literature, both full time-domain switched and simplified models have been proposed to simulate the behaviour of inverters. Switched models are very accurate, but computationally intensive, making this approach unfeasible for large system studies [1]. Simplified models on the other hand, drastically increase computation speed [2], [3]. The challenge here is to preserve the desired accuracy when developing the model. As different studies require different levels of accuracy, the simplified model, and consequently its achievable computation speed, depends on the objectives of the study. In this paper, the focus is on modelling the short-circuit behaviour of IIDG for protection system studies. Most IIDG use a voltage-source PWM inverter and therefore only this type of inverter is considered here. As both primary and backup protections are considered in protection studies, the models should approximate the actual behaviour of the inverter within a time range of ten milliseconds (ms) to a few seconds (the maximal fault clearing time in the Belgian Federal Grid Code is $3.1 \mathrm{~s}$ or lower, depending on the voltage level [4]).

The paper is organised as follows: Section II gives an overview of techniques reported in literature. Based on this report, the averaged value modelling technique (AVM technique) is selected. These dynamic averaged value models (AVMs) are further treated in section III: examples from literature are given and a case study compares the short-circuit behaviour and simulation time of a full time-domain switched model and its averaged value model (AVM).

\section{OVERVIEW OF SHORT-CIRCUIT SIMULATION TECHNIQUES}

Several simplified models for IIDG in power system simulations are proposed in literature, as it is generally accepted that full time-domain switched models are computationally too intensive or time consuming for large system studies [1]-[3], [5], [6]. Three groups of techniques are discussed: simple load flow (LF) based techniques, advanced LF based techniques that model the actual fault response and finally AVM techniques that simulate the behaviour of the control system of the inverter.

\section{A. Simple LF based techniques}

The first group that is discussed are load flow based techniques that make assumptions on the response of inverters to a fault in the network. In these models, the injected current during a fault is either fixed or depends on the voltage at the inverter terminals. In the former case a straightforward calculation is possible, in the latter case an iteration process is required as the injected current influences the voltages in the network.

The simplest technique, described in [7], considers the IIDG as a controlled current source and assumes the IIDG delivers its maximal fault current during faults. As the inverter controls are assumed to produce only positive sequence currents, an equivalent sequence component representation is suggested, where both the negative and zero sequence impedances are infinite. Standard short-circuit calculation techniques [8] are then used and a positive sequence reactance is calculated iteratively, so that the IIDG delivers its maximal fault current. If the calculation software is able to handle fixed current sources, the iteration process can be avoided. It is clear that this method is simple and that it allows calculating a safe estimate for the maximal fault current. However, in grids with a high share of IIDG, a fault is never close to all 
IIDG. Therefore not all IIDG inject their maximal current and this technique results in an overestimation of the total fault current. In addition, this technique does not provide an accurate evaluation of the network voltages, used in distance protection schemes.

Some sources [9], [10] propose a similar technique, but take into account that the maximal fault current is not the only parameter of importance and that, especially when considering protection settings, a more accurate method is required. Reference [9] proposes to use existing short-circuit analysis software, but to include a table lookup technique to relate the IIDG's injected current to the voltage at its terminals. The time dependency of the injected current can be taken into account in the tables, but this assumes that the voltage remains constant during the fault, which might not be a valid assumption in case of a high share of IIDG. An alternative is given in [10], where a mathematical relationship determines the injected current: a positive sequence reactive current in function of the positive sequence voltage drop below 0.9 p.u. Both [9] and [10] assume a specific control strategy and provide improved accuracy, but unlike [10], the table method of [9] does not take into account the operating point of the IIDG prior to the fault. Implicitly, a control strategy that switches to a voltage based strategy during voltage dips is assumed. However, for some IIDG like wind turbines, a transfer of active power may be required to enable fault ride through (FRT). This means that the injected current also depends on the operating point of the IIDG prior to the fault. In general, these methods have too many limitations to perform accurate protection studies in grids with a high share of IIDG.

\section{B. Advanced LF based techniques}

The second group are the load flow techniques that model the actual fault response by taking some elements of the control strategy into account. An advanced load flow technique for grids with IIDG is described in [11]. That paper does not aim to calculate short-circuit currents, but it proposes a load flow technique where operating limits are checked during the load flow calculation and various inverter control strategies are considered for steady state situations (e.g. negative sequence current injection).

More advanced techniques for short-circuit calculations are found in [2] and [5]. The former determines iteratively which inverters go into current limit mode during a shortcircuit, taking into account that most IIDG are operated as PQ sources and only switch to a current limiting strategy to protect the power electronic components in the inverter. This method does not simply assume that all inverters inject their maximal currents and is therefore able to calculate both voltages and currents during faults. Although different current limiting strategies are mentioned, only a predefined limit in the synchronously rotating reference frame is modelled, making this method only valid for this control strategy: e.g. it does not consider FRT active power transfer requirements during the fault. A positive sequence current source is proposed as the equivalent sequence component representation in the current limit mode to integrate the IIDG in the calculation procedure. Reference [5] continues on this work. Here, an additional fault model for a current limiting strategy in the natural reference frame is developed. This model responds differently to asymmetrical faults: in three leg inverters negative sequence currents can also be injected and in four leg inverters both negative and zero sequence currents are possible. As the focus of [5] is on stand-alone grids, with low short-circuit powers, it assumes that all inverters in the network go into current limiting mode as the voltage drops during faults are large in weak grids. Both [2] and [5] could be combined, but it is clear that the control and certainly the current limiting strategy have a large influence on the fault model that has to be used. For each control/current limiting strategy a different fault model has to be developed to obtain accurate simulation results.

\section{AVM techniques}

The third group are the AVM techniques. The IEEE Task Force on Dynamic Average Modelling has given a complete overview, with more than 100 references, of AVMs in [1]. They explain AVMs as an approximation of the original system by averaging the effect of fast switching within a prototypical switching interval. The key idea is that the discontinuity of the switched models can be avoided and that the number of time steps can be reduced, resulting in much faster simulations. Typical IIDG have a switching frequency of several $\mathrm{kHz}$ [1], [12]. A detailed switched model would require a time step that is considerably smaller to accurately calculate the variables during a switch cycle. In the AVM, one average value is defined over the length of a switching interval. For DC-AC converters (inverters), this cannot be applied directly according to [1], but a transformation to a synchronously rotating reference frame should be applied first. However, it is also possible to do the averaging in $\mathrm{AC}$ variables if the switching frequency is substantially higher than the output signal that is produced. This means that the signal that is modelled is considered as a DC signal (or low frequency signal) compared to the switching frequency. In [13] this approach is explained specifically for DC/AC half-bridge converters, two-level, three-phase voltage-sourced converters and three-level, three-phase, neutral-point clamped (NPC) voltagesourced converters. All AVMs have the advantage that they are continuous and capable of using much larger time steps than the switched, discontinuous models [1].

In this paper, the AVMs according to [13] are considered. The ideal version of this model consists of voltage sources $m_{i} \cdot V_{d c} / 2$ in each phase $(i=a, b, c)$, where the modulation indices $m_{i}$ are determined by the control system of the inverter. Additional elements can be added to include switching and conduction losses. The DC power balance can be included as well. Reference [3] mentions that the AVM does not depend on the converter topology or the modulation technique, but [14] is more careful in this regard, and states that, under simplifying assumptions, the model of the conventional two-level voltagesourced converter can be deduced from that of the three-level NPC. 
Another way to look at these models is mentioned in [15]: if the switching frequency is high enough and the output filter is designed correctly, the switching harmonics are attenuated. The frequency components of the reference voltage and the generated voltage of the inverter are then equal in the lower frequency range. As the AVMs model the low frequency variations accurately, they also take into account the low frequency behaviour of the control system. The control system of the inverter is simulated completely or in a simplified form to allow this.

There are however some drawbacks to AVMs. Compared to full time-domain switched models, information on high frequency components (e.g. harmonics) is lost [12]. Reference [12] states that the AVM is valid up to about one-third of the inverter switching frequency, while [16] states that the AVM is valid up to half of the switching frequency. In [15] an important condition is added: an AVM is only valid if the frequencies in the reference signal are much smaller than the resonance frequency of the filter. From the viewpoint of protection studies, the time range of interest ranges from about ten milliseconds (ms) to a few seconds. The AVMs preserve most dynamics in this time range and they provide sufficient accuracy for protection studies. Compared to the LF based techniques, the simulations are slower as a time based simulation is required. This time based simulation is however significantly faster than the simulation of the switched model.

\section{Choice of a suitable simulation technique}

1) Different control strategies: As mentioned throughout the discussion of the techniques, there are several control strategies for IIDG. A good overview of the wide range of possible control strategies for IIDG is given in [17]: three choices exist for the control reference frame (synchronous reference $(d q)$, stationary reference $(\alpha \beta)$ or natural reference $(a b c)$ frame) and four major control strategies for IIDG under grid faults are discussed: unity power factor, positive sequence, constant active power and constant reactive power control strategy. Because there are so many control strategies and they have a significant impact on the injected currents during faults, it seems appropriate to incorporate the most important aspects of the control system of the inverters in the simulation models. This is certainly important if one wants to investigate the influence of these different control strategies on the protection system. As is mentioned in [18], the current control strategy (which is the fast acting inner loop of the control system) even influences the fault response during the first period of a single-phase grid fault behind the connection transformer of an IIDG.

The first group of techniques does not take the control strategy into account or assumes a simple control strategy. In general, these techniques are not sufficiently accurate if the grids that are studied contain a high share of IIDG. The second group of techniques takes some elements of the control system into account. However, every different control strategy of the inverter requires the development of a new equivalent model if the approach of [2] and [5] is used. In addition, [10] states that the zero- and negative sequence responses of inverters are a design parameter and that there is no corresponding sequence representation. In general, this means that the simulation model of the IIDG should include its control strategy. AVMs are well-suited for this task.

2) Flexibility of modelling and simulation efficiency: Although the AVMs are significantly faster than the switched models, they are slower than the LF based techniques. Therefore, it seems logical to investigate whether a simplified model, similar to the techniques in [2] and [5], can be developed. First, the different control strategies for inverters during faults, and their impact on the protection systems of grids with a high share of IIDG, should be investigated. Once the optimal control strategy is determined, such a simplified model can be developed if the model includes the relevant parts of the control strategy. As there is no general consensus on the optimal control strategy for inverters during fault situations and several new control strategies have been proposed in recent years [17], the grid standards have changed and will probably change again in the future when new issues are identified. Therefore, the authors want to focus on the AVMs for protection studies as these AVMs enable to incorporate the control system of the inverter in a straightforward way. Investigating another control strategy requires minimal changes to the simulation model, as only the new inverter control strategy has to be modelled.

As explained in section II-C, the AVMs offer an increased efficiency of the simulations compared to the switched models. The next section illustrates this. This increased efficiency is required to simulate grids with a high share of IIDG, as each inverter that would be added with a switched model would unnecessarily increase the complexity of the simulation, resulting in unfeasible simulation times for large systems.

\section{ILLUSTRATION OF AVMS IN POWER SYSTEM STUDIES}

In this section, examples of AVMs found in literature are mentioned. Consecutively a comparison is made between the fault responses of a switched model and its AVM.

\section{A. Results from literature}

There are several examples of AVM applications in literature. Three examples are mentioned here briefly.

Reference [3] describes AVMs for HVDC transmission systems with voltage-sourced converters. The models are similar to the approach described in section II-C. The AVMs are reported to give an accurate replication of the dynamic behaviour of the IGBT based switched models. Simulation examples in [3] show that AVMs give a significant gain in simulation time: gains of more than 20 times are easily reached. Another example of AVMs is found in [19]: here an AVM is applied to increase the simulation speed of a doubly-fed induction generator (DFIG). The AVM that is used for the voltagesourced converter feeding the rotor circuit is again similar to the AVM described in section II-C. The simulation gains that are reached depend strongly on the induction machine model, but it is clear that the AVM enables a serious reduction in simulation time. The last example of an AVM application is 


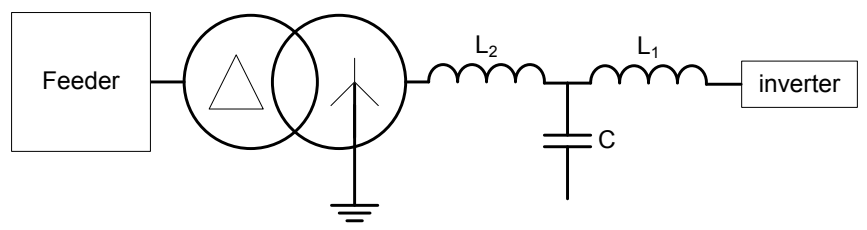

Fig. 1. Grid model

TABLE I

GRID DATA

\begin{tabular}{c|c|c}
\hline Element & Property & Value \\
\hline System feeder & Rated voltage (L-L) & $15 \mathrm{kV}$ \\
& Short-circuit power & $200 \mathrm{MVA}$ \\
& X/R ratio & 10 \\
& Frequency & $50 \mathrm{~Hz}$ \\
\hline Transformer & Rating & $2.5 \mathrm{MVA}$ \\
& Voltage ratio & $15 \mathrm{kV} / 400 \mathrm{~V}$ \\
& Connection & Dy11 (solidly earthed) \\
& Impedance voltage & $6 \%$ \\
\hline Inverter & Rating & $60 \mathrm{kVA}$ \\
& DC voltage & $800 \mathrm{~V}$ (constant) \\
& Carrier frequency & $10 \mathrm{kHz}$ \\
\hline Filter & $L_{1}$ & $2.3 \mathrm{mH}$ \\
& $L_{2}$ & $0.9 \mathrm{mH}$ \\
& $C$ & $8.8 \mu \mathrm{F} \mathrm{(star} \mathrm{connected)}$
\end{tabular}

found in [12]: here the AVMs are mentioned as an alternative to switched models for faster simulations in power system studies of three-phase PV systems.

\section{B. Example of fault response}

1) Simulation model: To compare the fault response of a switched model and its AVM, a simulation model is built in Matlab/Simulink with the PLECS Blockset [20]. The electrical part of the model (grid and inverter) is modelled in PLECS and the inverter control system in Simulink. The PLECS configurable subsystems allow to create one model, with one Simulink control system, and to select the appropriate configuration for the inverter: switched or AVM. The grid model is shown in Fig. 1. It consists of a system feeder, modelled as a voltage source and an impedance, a delta-star transformer and a low voltage inverter unit with an LCL filter. The grid data is shown in Table I. The switched model is the standard three-phase, two-level IGBT converter model of PLECS. Both the IGBT and the reverse diodes are ideal in this model. PLECS needs only six internal switches to represent this converter, so the simulation of this model is already faster

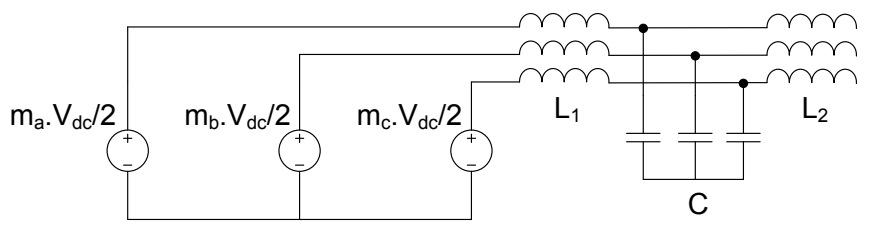

Fig. 2. AVM and filter of the simulation model

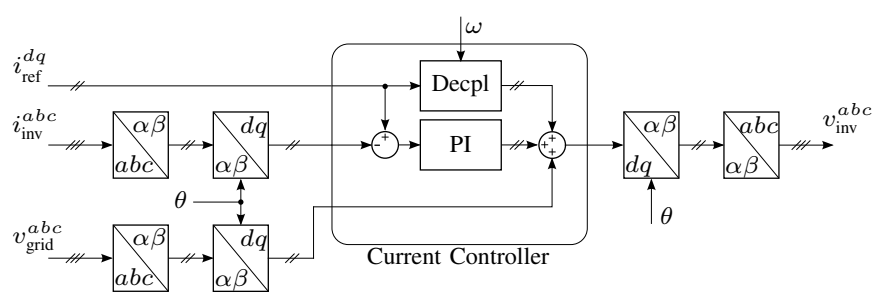

Fig. 3. Current control loop in the synchronous $d q$ reference frame.

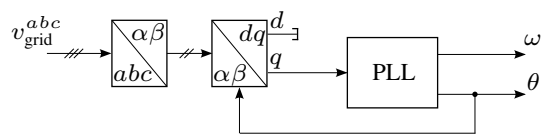

Fig. 4. Basic PLL for grid synchronisation.

than the simulation of a model that specifies all electrical parameters of the IGBT and diodes. The AVM is shown in Fig. 2 and consists of voltage sources $m_{i} \cdot V_{d c} / 2$ in each phase ( $i=a, b, c)$, where the modulation indices $m_{i}$ are controlled by the inverter control system in Simulink. This model is based on the averaged ideal three-phase voltage-sourced converter that is explained in detail in [13]. The DC bus voltage of the inverter is modelled as a constant DC voltage source. As can be seen in Fig. 2, the AVM only replaces the switched model, the filter is not included in the AVM.

2) Control strategy: The control system consists of a current control loop in the synchronous $d q$ reference frame, and a phase-locked loop (PLL). The current references are kept fixed, and are therefore independent of the voltages in the system. This simplification allows to compare the fault responses of the switched model and its AVM without having to develop a current limiting strategy. Other papers, e.g. [21], give more information on setting current references during grid faults.

A schematic diagram of the current control loop is shown in Fig. 3. In this figure, $i_{\text {ref }}$ is the current reference, $i_{i n v}$ is the current measured at the output of the inverter (before the filter), $v_{\text {grid }}$ is the voltage measured at the grid side of the inverter (after the filter) and $v_{i n v}$ is used to determine the modulation indices $m_{i}$ of the inverter (switched model or its AVM). The superscripts indicate the reference frame in which the values are expressed. The $d q$ decoupling (Decpl) terms, and grid voltage feedforward term are added after the PI controller. The grid voltage phase angle, required to transform the three-phase variables $a b c$ to the synchronous reference frame variables $d q$, is estimated with a PLL.

Two PLL configurations are considered. In the first configuration, shown in Fig. 4, the PLL drives the $q$-component of the measured grid voltage to zero with a PI controller. This PLL configuration does not take into account (unbalanced) fault situations. Several sources, e.g. [22], [23], stress the importance to detect the positive and negative sequence voltages separately under unbalanced grid conditions. Therefore, a second, more advanced, PLL configuration was constructed as shown in Fig. 5. In a first step, the fundamental frequency 


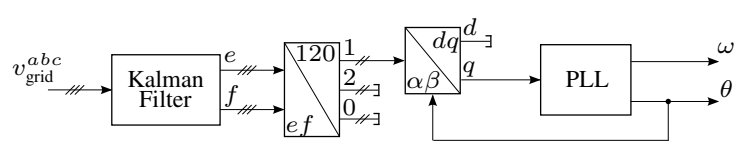

Fig. 5. Advanced PLL for grid synchronisation.

components of the $a b c$ line-to-neutral voltages are determined with a Kalman estimator, as was set forth in [24] for single phase applications. These voltages are then transformed into positive, negative and zero sequence components with the help of transformations similar to [25]:

$$
\left[\begin{array}{l}
e_{1} \\
f_{1} \\
e_{2} \\
f_{2} \\
e_{0} \\
f_{0}
\end{array}\right]=\frac{1}{3} \frac{\sqrt{3}}{\sqrt{2}}\left[\begin{array}{cccccc}
1 & 0 & -\frac{1}{2} & -\frac{\sqrt{3}}{2} & -\frac{1}{2} & \frac{\sqrt{3}}{2} \\
0 & 1 & \frac{\sqrt{3}}{2} & -\frac{1}{2} & -\frac{\sqrt{3}}{2} & -\frac{1}{2} \\
1 & 0 & -\frac{1}{2} & \frac{\sqrt{3}}{2} & -\frac{1}{2} & -\frac{\sqrt{3}}{2} \\
0 & 1 & -\frac{\sqrt{3}}{2} & -\frac{1}{2} & \frac{\sqrt{3}}{2} & -\frac{1}{2} \\
1 & 0 & 1 & 0 & 1 & 0 \\
0 & 1 & 0 & 1 & 0 & 1
\end{array}\right] \cdot\left[\begin{array}{c}
e_{a} \\
f_{a} \\
e_{b} \\
f_{b} \\
e_{c} \\
f_{c}
\end{array}\right]
$$

where $e_{a, b, c}$ are the fundamental frequency components and $f_{a, b, c}$ are their quadrature components as determined by the Kalman estimator. This is an application of the instantaneous symmetrical components expressed with a $90^{\circ}$ phase shift, as described in [26]. Because the quadrature signal lags the signal, the signs in the equation mentioned here are different for the quadrature components. The factor $\frac{\sqrt{3}}{\sqrt{2}}$ arrives from the equivalence with the control system variables $\alpha \beta$ in this specific implementation. The positive sequence components $e_{1}$ and $f_{1}$ are then used instead of the $\alpha \beta$ signals to determine the phase angle reference in the PLL. The current control loop remains unchanged. This PLL configuration is referred to as the advanced PLL, the other PLL configuration is referred to as the basic PLL in this paper.

3) Simulation results: Several fault types have been simulated. For all fault types the AVM fault response is a good approximation of the switched model fault response. To limit the number of plots and focus on the comparison of the switched model and its AVM, only results for the three and two-phase fault (between phase $b$ and $c$ ) are shown. The fault always occurs at the low voltage side of the transformer at $t=0.5 \mathrm{~s}$

Fig. 6 shows the fault response of the inverter to a threephase fault. The basic PLL configuration is used, but as a three-phase fault results in a balanced situation, this does not result in problems. The voltages at the low voltage (LV) and medium voltage (MV) side of the transformer are shown in Fig. 6a-6b. The other figures compare the switched model (on the left) with its AVM (on the right). The current responses of both models are very similar. As was mentioned previously, the current reference is kept fixed in the control system. This explains why the current does not increase during the fault.

Next, a two-phase fault was applied to the same system. The results are shown in Fig. 7, which shows the same order of plots as Fig. 6. Again the current responses of both models are very similar. Fig. 8 shows zoomed parts of Fig. 7. The switched model and its AVM are plotted on top of each other

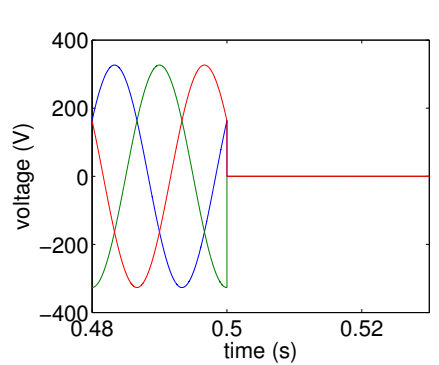

(a)

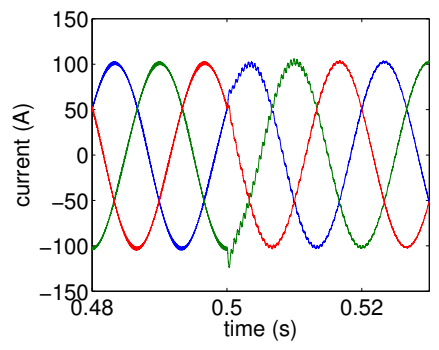

(c)

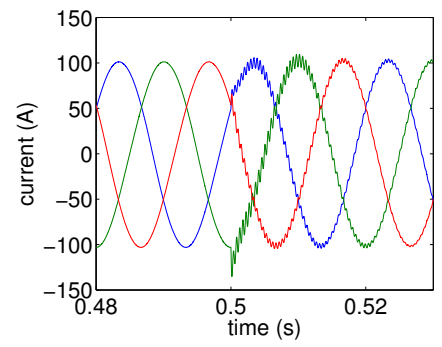

(e)

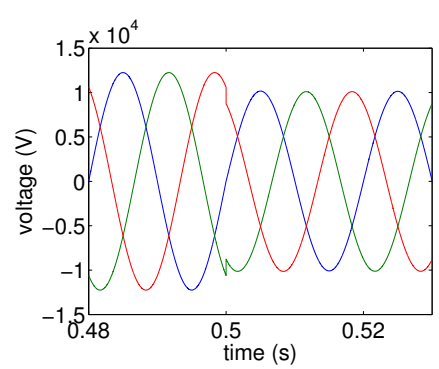

(b)

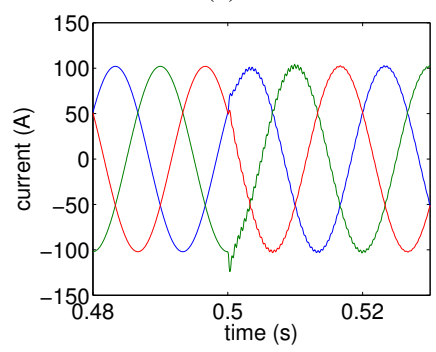

(d)

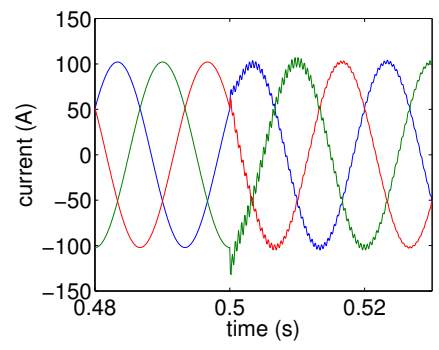

(f)
Fig. 6. Comparison between the switched model and its AVM for a threephase fault, with the basic PLL configuration: (a) Voltage at the LV side and (b) at the MV side of the transformer. (c) Inverter output current (before the filter) of the switched model and (d) its AVM. (e) Inverter output current (after the filter) of the switched model and (f) its AVM.

to show their close resemblance. Fig. $8 \mathrm{a}-8 \mathrm{~b}$ show the current in phase $a$ before and after the filter. Since the current after the filter is the most relevant for protection studies, the phases $b$ and $c$ are shown in Fig. 8c-8d. It is clear from these figures that the basic PLL is not able to accurately track the phase angles of the voltages. This is because the negative sequence voltages appear as second order harmonics after the transformation from $a b c$ to $d q$ coordinates [17]. The basic PLL is not able to deal with these second order harmonics and the phase angle reference becomes distorted. The AVM accurately reproduces this as it is an effect related to the voltages in the grid during the fault. This problem and solutions to track the phase angles of the voltages during unbalanced faults, are explained in detail in [27]. The main idea is to track the positive sequence and negative sequence separately. This is done in the advanced PLL as explained before. Subsequently this PLL configuration has been included in the simulation models and the same two-phase fault was applied to the system at the same time. Again, the switched model is shown next to its AVM in Fig.9. Because the inverter is small, it does not noticeably influence the voltages in the system, as can be seen by comparing the voltages during the fault in Fig. 7 and 9. But as expected 


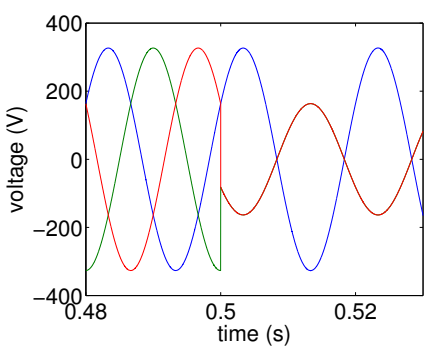

(a)

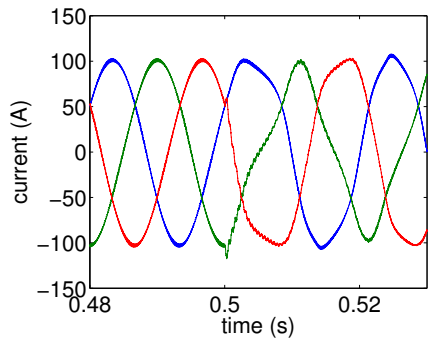

(c)

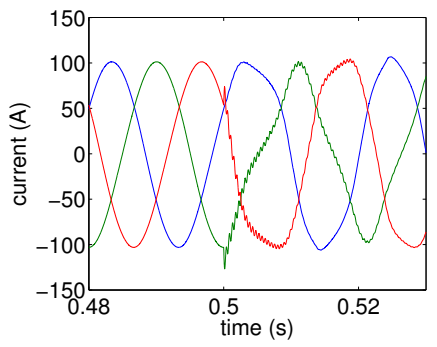

(e)

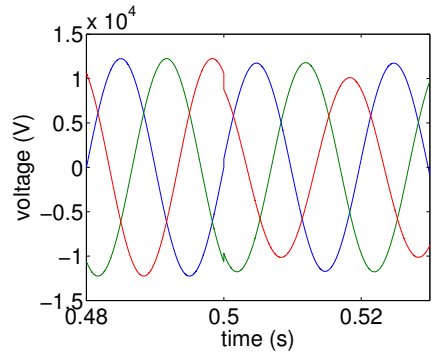

(b)

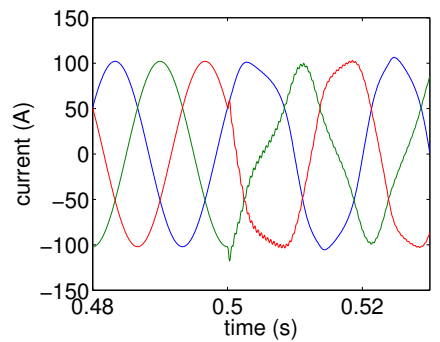

(d)

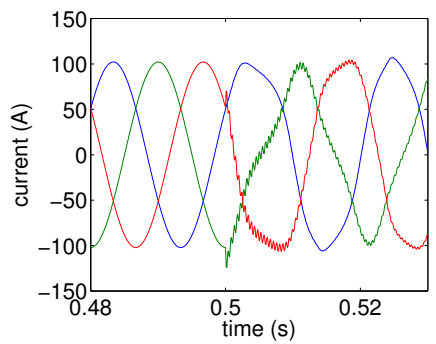

(f)

Fig. 7. Comparison between the switched model and its AVM for a twophase fault (phase $b$ and $c$ ), with the basic PLL configuration: (a) Voltage at the LV side and (b) at the MV side of the transformer. (c) Inverter output current (before the filter) of the switched model and (d) its AVM. (e) Inverter output current (after the filter) of the switched model and (f) its AVM.

the inverter control system responds differently to the fault: the currents are not deformed and they are hardly influenced by the fault because the current reference is unaltered. Again the AVM fault response closely resembles the switched model fault response. This illustrates that the AVM simulates the control system and allows to investigate the influence of the inverter control systems in grids with a high share of IIDG.

The techniques that are applied are generic, so they are also valid for larger IIDG with voltage-source PWM inverters, connected to the medium voltage grid. These units often have lower switching frequencies. From the discussion of AVMs in section II-C, it is clear that the AVM will differ more from the switched model in this case. But in general the switching frequencies are higher than $1000 \mathrm{~Hz}$ (20 times the fundamental frequency in $50 \mathrm{~Hz}$ grids) and AVMs give a good approximation. As an illustration, the simulation of a twophase fault with the basic PLL configuration was repeated with a switched model of $5000 \mathrm{~Hz}$. The filter is the same as in the previous model, although in principle a different switching frequency requires a redesign of the LCL-filter. The inverter output current of the switched model is plotted in Fig. 10 next to its AVM, which is of course the same as the AVM of Fig. 7.
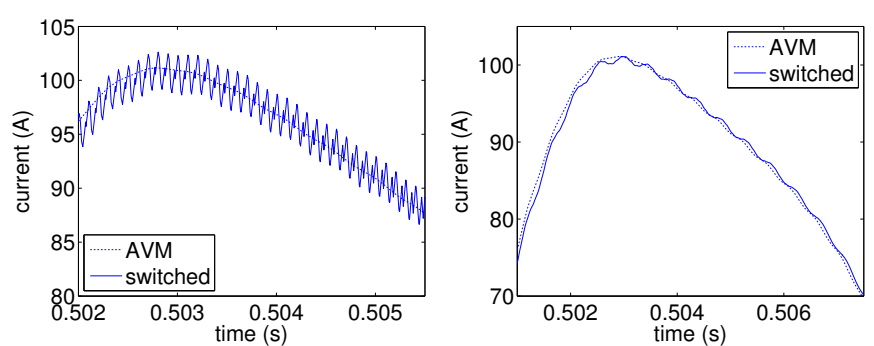

(a)

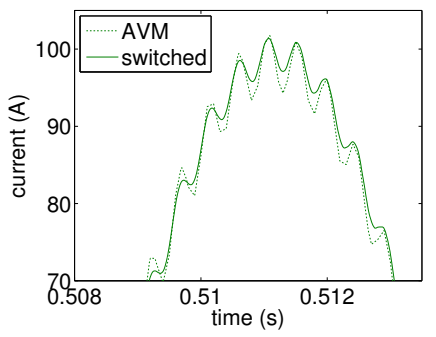

(c)

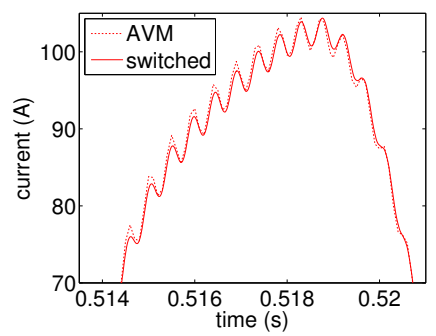

(d)

Fig. 8. Detailed comparison between the switched model and its AVM for a two-phase fault (phase $b$ and $c$ ), with the basic PLL configuration: (a) Inverter output current (before the filter). (b), (c) and (d) Inverter output current (after the filter) of the three phases.

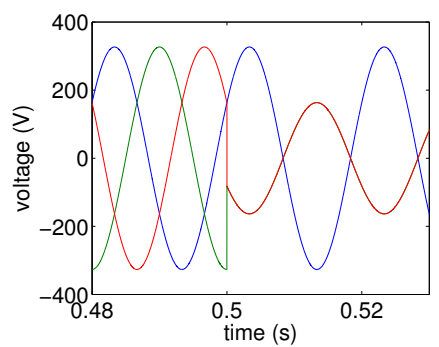

(a)

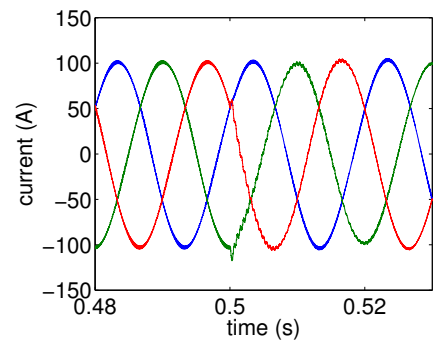

(c)

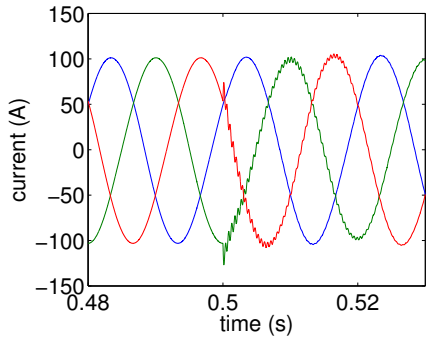

(e)

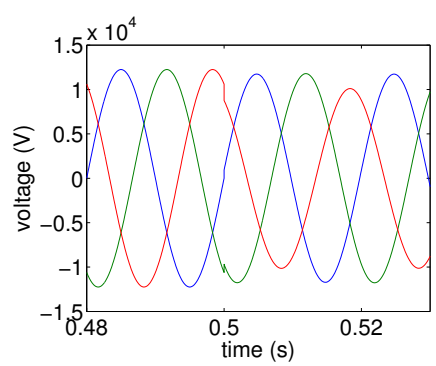

(b)

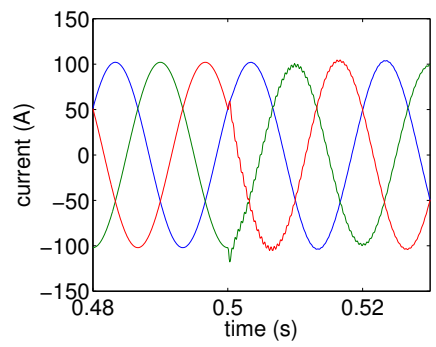

(d)

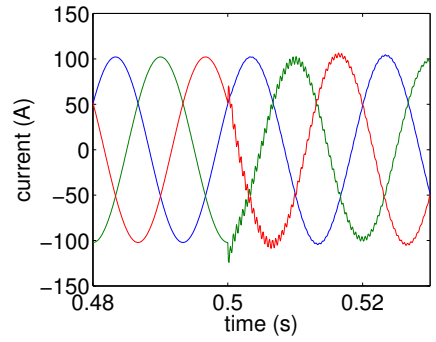

(f)
Fig. 9. Comparison between the switched model and its AVM for a twophase fault (phase $b$ and $c$ ), with the advanced PLL configuration: (a) Voltage at the LV side and (b) at the MV side of the transformer. (c) Inverter output current (before the filter) of the switched model and (d) its AVM. (e) Inverter output current (after the filter) of the switched model and (f) its AVM. 


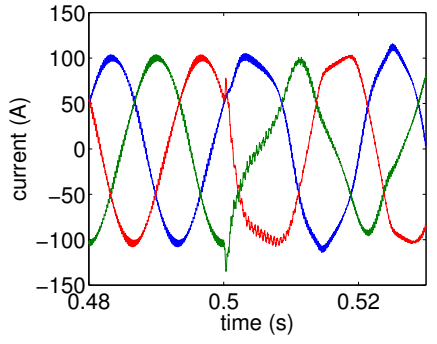

(a)

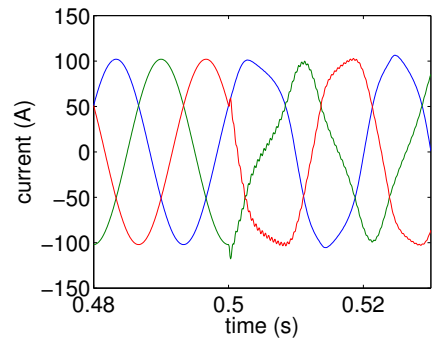

(b)
Fig. 10. Comparison between the switched model and its AVM for a twophase fault (phase $b$ and $c$ ), with the basic PLL configuration: (a) Inverter output current (before the filter) of the switched model at $5000 \mathrm{~Hz}$ and (b) of its AVM.

All examples illustrate that AVMs give a very good approximation of the fault response of the switched models. Only when the higher harmonics are important, e.g. when the switching frequency harmonics interact with other parts of the system (e.g. resonance), the AVMs are not sufficiently accurate. For a properly designed IIDG, this is not the case and the AVM technique is then a suitable technique for protection studies.

4) Simulation speed: For the basic PLL configuration, simulating the AVM for 3 seconds required around $11.8 \mathrm{~s}$, while the switched model required around $194 \mathrm{~s}$. This simulation time was chosen because it is around the maximal time that is required in protection studies (see section I). For the advanced PLL configuration the AVM simulation takes about $13.1 \mathrm{~s}$, compared to about $225 \mathrm{~s}$ for the switched model. The AVM is thus significantly faster (factor 16-17) than the switched model, although the same computer was used, and both models used the same Simulink control system. These gains in simulation speed are similar to the gains reported in [3]: the gains are a bit smaller, but in [3] the comparison is made with a switched model that contains non-ideal diodes and a snubber circuit. Here the PLECS switched model only contains ideal diodes.

\section{COnClusions}

This paper gives an overview of calculation techniques for short-circuit currents and voltages in grids with a high share of IIDG. It is shown that the simplest techniques that don't take the control strategy into account are too limited when detailed protection studies are required. The advanced load flow based techniques are shown to be very promising, but they lack the ability to easily model any given control system of the IIDG. Therefore, the AVM technique is proposed for detailed protection studies. As stated in literature, the simulation time is drastically reduced compared to full time-domain switched models, but AVMs still accurately model the control behaviour of the inverters. This is verified by comparing the fault responses of both models in a case study. The AVM technique will be used in future research on protection systems in grids with a high share of IIDG.

\section{ACKNOWLEDGMENT}

Both T. Wijnhoven and J. Tant have a Ph. D. fellowship of the Research Foundation - Flanders (FWO) and wish to acknowledge the financial support of the FWO.

\section{REFERENCES}

[1] S. Chiniforoosh, J. Jatskevich, A. Yazdani, V. Sood, V. Dinavahi, J. A. Martinez, and A. Ramirez, "Definitions and Applications of Dynamic Average Models for Analysis of Power Systems," IEEE Transactions on Power Delivery, vol. 25, no. 4, pp. 2655-2669, 2010.

[2] C. A. Plet, M. Graovac, T. C. Green, and R. Iravani, "Fault response of grid-connected inverter dominated networks," in IEEE Power and Energy Society General Meeting, 2010, pp. 1-8.

[3] J. Peralta, S. Dennetiere, and J. Mahseredjian, "Average-value Models for the Simulation of VSC-HVDC Transmission Systems," in Cigré Int. Symp. The Electric Power System of the Future, Integrating supergrids and microgrids, Bologna, Italy, 2011.

[4] "Royal Decree of 19 December 2002: Federal Grid Code Belgium," Belgisch staatsblad 28.12.2002, pp. 58 471-58541.

[5] C. Plet, M. Brucoli, J. McDonald, and T. Green, "Fault models of inverter-interfaced distributed generators: Experimental verification and application to fault analysis," in IEEE Power and Energy Society General Meeting, 2011, pp. 1-8.

[6] S. Achilles and M. Pöller, "Direct drive synchronous machine models for stability assessment of wind farms," in 4th International Workshop on Large Scale Integration of Wind Power and Transmission Networks for Offshore Wind-Farms, Billund, Denmark, 2003, pp. 1-9.

[7] M. Fischer and A. Mendonça, "Representation Of Variable Speed Full Conversion Wind Energy Converters For Steady State Short-Circuit Calculations," in IEEE Power and Energy Society General Meeting, 2011, pp. 1-7.

[8] G. Balzer, D. Nelles, and C. Tuttas, Kurzschlussstromberechnung nach VDE 0102. VDE-Verlag, 2001.

[9] R. A. Walling, E. Gursoy, and B. English, "Current Contributions from Type 3 and Type 4 Wind Turbine Generators During Faults," in IEEE Power and Energy Society General Meeting, 2011, pp. 1-4.

[10] R. J. Nelson and H. Ma, "Short-Circuit Contributions of Full-Converter Wind Turbines," in IEEE Power and Energy Society General Meeting, 2011, pp. 1-4.

[11] M. Z. Kamh and R. Iravani, "A Unified Three-Phase Power-Flow Analysis Model For Electronically Coupled Distributed Energy Resources," IEEE Transactions on Power Delivery, vol. 26, no. 2, pp. 899-909, Apr. 2011.

[12] A. Yazdani, A. R. Di Fazio, H. Ghoddami, M. Russo, M. Kazerani, J. Jatskevich, K. Strunz, S. Leva, and J. A. Martinez, "Modeling Guidelines and a Benchmark for Power System Simulation Studies of Three-Phase Single-Stage Photovoltaic Systems," IEEE Transactions on Power Delivery, vol. 26, no. 2, pp. 1247-1264, Apr. 2011.

[13] A. Yazdani and R. Iravani, Voltage-Sourced Converters in Power Systems. John Wiley and Sons, 2010.

[14] _ "A Generalized State-Space Averaged Model of the Three-Level NPC Converter for Systematic DC-Voltage-Balancer and CurrentController Design," IEEE Transactions on Power Delivery, vol. 20, no. 2, pp. 1105-1114, Apr. 2005.

[15] J. Morren, S. W. H. de Haan, and J. A. Ferreira, "Model reduction and control of electronic interfaces of voltage dip proof DG units," in IEEE Power Engineering Society General Meeting, 2004, pp. 2168 2173 Vol.2.

[16] M. Cespedes, T. Beechner, and J. Sun, "Averaged modeling and analysis of multilevel converters," in IEEE 12th Workshop on Control and Modeling for Power Electronics (COMPEL), 2010, pp. 1-6.

[17] F. Blaabjerg, R. Teodorescu, M. Liserre, and A. Timbus, "Overview of control and grid synchronization for distributed power generation systems," IEEE Transactions on Industrial Electronics, vol. 53, no. 5, pp. 1398-1409, 2006.

[18] A. Timbus, M. Liserre, R. Teodorescu, P. Rodriguez, and F. Blaabjerg, "Evaluation of Current Controllers for Distributed Power Generation Systems," IEEE Transactions on Power Electronics, vol. 24, no. 3, pp. 654-664, Mar. 2009. 
[19] J. Morren, S. de Haan, P. Bauer, J. Pierik, and J. Bozelie, "Comparison of complete and reduced models of a wind turbine with Doubly-Fed Induction Generator," in Proc. 10th European Conference Power Electronics Applications (EPE), Toulouse, France, 2003, pp. 1-10.

[20] Plexim GmbH, "PLECS Blockset." [Online]. Available: http://www.plexim.com/

[21] P. Rodriguez, A. Timbus, R. Teodorescu, M. Liserre, and F. Blaabjerg, "Flexible Active Power Control of Distributed Power Generation Systems During Grid Faults," IEEE Transactions on Industrial Electronics, vol. 54, no. 5, pp. 2583-2592, Oct. 2007.

[22] G. Saccomando and J. Svensson, "Transient operation of grid-connected voltage source converter under unbalanced voltage conditions," in IEEE 36th Industry Applications Society (IAS) Annual Meeting, vol. 4, 2001, pp. 2419-2424.

[23] P. Rodriguez, A. Luna, M. Ciobotaru, R. Teodorescu, and F. Blaabjerg, "Advanced Grid Synchronization System for Power Converters under Unbalanced and Distorted Operating Conditions," in IEEE 32nd Annual Conference on Industrial Electronics (IECON), Nov. 2006, pp. 5173-5178.

[24] K. De Brabandere, T. Loix, K. Engelen, B. Bolsens, J. Van den Keybus, J. Driesen, and R. Belmans, "Design and Operation of a Phase-Locked Loop with Kalman Estimator-Based Filter for SinglePhase Applications," in IEEE 32nd Annual Conference on Industrial Electronics (IECON), Nov. 2006, pp. 525-530.

[25] B. Bolsens, K. De Brabandere, J. Van den Keybus, J. Driesen, and R. Belmans, "Three-Phase Observer-based Low Distortion Grid Current Controller Using an LCL Output Filter," in IEEE 36th Power Electronics Specialists Conference (PESC), 2005, pp. 1705-1711.

[26] M. Iravani and M. Karimi-Ghartemani, "Online estimation of steady state and instantaneous symmetrical components," IEE Proceedings Generation, Transmission and Distribution, vol. 150, no. 5, p. 616, 2003.

[27] R. Teodorescu, M. Liserre, and P. Rodriguez, Grid Converters for Photovoltaic and Wind Power Systems. John Wiley and Sons, 2011. 